\title{
LA ORIENTACIÓN FAMILIAR COMO EXPERIENCIA PEDAGÓGICA
}

Gabriel Castellanos Bernal

LA ORIENTACIÓN FAMILIAR ES EL CONJUNTO DE CONTENIDOS, RECURSOS Y procedimientos para apoyar la tarea educativa en la familia. Es innegable la necesidad de esta orientación en una sociedad que plantea nuevos retos y exigencias a los padres, para quienes son insuficientes unas cuantas nociones de educación general. Ahora ellos necesitan aprender con profundidad conceptos de educación familiar, a fin de mantener la unidad y la superación de quienes integran la familia.

En este artículo presentamos algunos aspectos de la actuación del pedagogo como orientador familiar. Su formación profesional, con enfoque humanista, le permite fundamentar teóricamente el trabajo que realiza con padres de familia. Les ayuda a cumplir el compromiso de educar con responsabilidad a sus hijos, y de crear y mantener un ambiente familiar lo más funcional posible.

\section{INTRODUCCIÓN}

Los cambios científicos, tecnológicos e ideológicos ocurridos en nuestra época han originado modificaciones importantes en diferentes ámbitos de la sociedad. El impacto se ha dejado sentir especialmente en la familia, donde padre, madre e hijos son principales protagonistas del quehacer educativo.

La sociedad cambia con el advenimiento de ideologías que afectan el pensamiento y la actuación del ser humano. También se pretende que la familia cambie: o se adapta o muere. Antes, la familia se basaba más en la autoridad del padre, era 
más procreativa, colaboraba en la producción económica y estaba más integrada a la sociedad. Actualmente los cónyuges tienen derechos jurídicos iguales, disminuye la natalidad, la familia es más consumista y peligra su estabilidad social.

Surgen estilos familiares anormales que se alejan del modelo tradicional. Por ejemplo, matrimonios breves de parejas románticas que vivirán juntos hasta que el divorcio los separe, unión libre de quienes argumentan que no necesitan papeles para garantizar su amor, matrimonios por complicidad que se casan con la condición de no tener hijos, ya que son peligrosos para su tranquilidad. Hogares monoparentales por abandono de uno de los cónyuges o porque él o ella decidió tener un hijo con alguien, pero sin la convivencia matrimonial.

Existe un grupo que pretende legalizar "matrimonios de homosexuales" y su derecho de adopción. También se utiliza la reproducción artificial como alternativa de la unión conyugal para perpetuar la especie.

Sin embargo, a pesar de estas anomalías, "la familia conserva características de espontaneidad, orden y cohesión" que la mantiene como célula básica de la sociedad. Además, las acciones en pro de la familia van en aumento: 1994 fue declarado Año Internacional de la Familia. Encontramos matrimonios que rechazan el divorcio y buscan solución a sus problemas recurriendo al orientador o al terapeuta familiar. Se crean más escuelas para padres de familia. Surgen nuevas estrategias de intervención con base en diferentes teorías psicológicas: gestalt, racional emotiva, sistémica, cognitiva, logoterapia, etcétera. Existe el apoyo de la Pastoral Familiar, por iniciativa de la Iglesia Católica. La pedagogía contribuye a la formación de profesionistas dedicados a la orientación familiar

Urge promover la orientación familiar en este momento histórico. Los padres necesitan conocer y contrarrestar aquellas influencias que deterioran su papel educativo. Es imprescindible resaltar la realidad de lo que constituye la familia 
como grupo humano y como sistema de comunicación interpersonal ${ }^{2}$. Necesitamos aprovechar los beneficios de la orientación familiar y aplicarlos con el optimismo que debe ponerse para solucionar problemas serios. Sabemos que la familia merece el más profundo respeto, porque en ella el ser humano encuentra el ambiente más adecuado para crecer como persona.

La atención a la familia se logra, entre otros medios, a través de programas de orientación familiar, cuyo propósito es elevar la calidad de vida en familia. La orientación familiar se utiliza como estrategia de intervención pedagógica para aumentar la capacidad educativa de los padres de familia.

\section{PAPEL DEL PEDAGOGO}

Respecto a la educación familiar es importante el papel del pedagogo, ya que está en condiciones de participar en el diseño, realización y evaluación de programas de orientación familiar. Por su formación profesional, el pedagogo tiene conocimientos acerca de la naturaleza de la familia, de sus funciones específicas y del papel que le corresponde en la dinámica social. Además, sus conocimientos del desarrollo humano, relaciones interpersonales, teorías de la personalidad, proceso y dinámica de grupos, procesos de aprendizaje, orientación personal y familiar; filosofía de la educación, antropología filosófica, y otros más le permiten fundamentar teóricamente la práctica pedagógica con padres de familia.

Se justifica la participación del pedagogo en el ámbito familiar, porque contribuye a la modificación de conceptos y habilidades de personas que sabrán afrontar, con dignidad humana, situaciones alegres o adversas de la vida real, tanto en la familia como en la sociedad.

Su trabajo principal consiste en la conducción de grupos de matrimonios en sesiones de orientación familiar, aplicando la metodología participativa; él es un facilitador del aprendizaje. 
También proporciona consultoría personal, matrimonial y familiar a quienes la soliciten.

\section{MARCO DE REFERENCIA}

La orientación familiar forma parte de un proceso educativo, que necesita bases teóricas para apoyar la parte práctica del mismo. Pretende capacitar a los padres para que cumplan responsablemente sus funciones de educadores, contando con la participación efectiva de los hijos y la oportuna intervención de otros parientes. Todos se benefician directamente con la ayuda recibida. Así, la orientación familiar requiere de una teoría que se fundamente en la dignidad de la persona humana y de todo lo humano.

El punto de vista que se adopte es de vital importancia, porque del concepto que se tenga de persona dependerá el trato que se dé al hombre respecto a su educación. Los recursos y procedimiento que se utilicen, las observaciones de la conducta y los métodos aplicados en el proceso de orientación estarán influidos por la teoría que sustente el pedagogo.

La pedagogía se ha enriquecido, conceptual y prácticamente, gracias a las aportaciones de teóricos ocupados en investigar el comportamiento humano desde diferentes perspectivas. Como ejemplo de teorías tenemos la psicodinámica, la conductista y la humanística. Cada una de ellas ha influido significativamente en el quehacer educativo.

El pedagogo tiene información suficiente acerca del método psicoanalítico de Sigmund Freud. También conoce el modelo conductista creado por John Watson y sistematizado por Frederic B. Skinner. Sin embargo, para el ejercicio de la orientación familiar, el pedagogo utiliza como principal marco de referencia el enfoque humanístico, que contempla al ser humano como un sujeto libre, responsable y comprometido con el desarrollo de su personalidad; es decir, de su propio perfeccionamiento.

Autores como Maslow, Rogers y Frankl, entre otros, sostie- 
nen un punto de vista humanístico. Ellos "han intentado formular teorías de la naturaleza humana que se basan en atributos humanos y problemas de existencia, características ocasionadas por la condición de ser humano,. Han rechazado los modelos típicos usados por psicólogos que representan a los seres humanos en términos biológicos o mecánicos”3. A continuación presentamos algunas ideas que sustentan estos autores.

Abraham Maslow confía en que el ser humano es capaz de lograr una personalidad madura y de mejorar el mundo en que vivimos. Le concede especial importancia a la motivación básica, pero su interés principal está centrado en las necesidades y motivos más elevados, aquellos que son claramente humanos.

Resulta muy conocida la escala de necesidades que Maslow elaboró para explicar la motivación humana. Subdivide las necesidades en básicas y de desarrollo o meta, ordenadas jerárquicamente: Fisiológicas, de seguridad, de amor y pertenencia, y de estima. Al quedar satisfechas estas necesidades básicas o inferiores, la persona experimenta la necesidad de autorrealización, que significa satisfacer la identidad personal en todos sus aspectos.

Carl Rogers es un psicólogo interesado en el desarrollo plenamente funcional del ser humano. Expresa su profunda empatía por toda la humanidad y, mediante su terapia centrada en el cliente, busca el bienestar de las personas. Sostiene que la falta de equilibrio y armonía psicológica se deben a la diferencia entre el concepto del yo ideal y el yo real, y que la solución está en la congruencia y conocimiento de sí mismo a fin de lograr una personalidad saludable ${ }^{4}$.

Rogers utiliza el concepto de respeto positivo para explicar que toda persona necesita aceptación, respeto y amor de los demás; porque dar y recibir respeto incondicional influye favorablemente en la conducta. En cambio el castigo injusto, el rechazo y el maltrato hacen la vida desagradable. Opina que a las personas se les acepta por lo que son, sin imponer condiciones 
que la hagan aceptable. Puede rechazarse la conducta negativa, pero la persona queda a salvo.

Viktor Frankl fundó una psicoterapia que rebasa las áreas fisiológica y afectiva de la personalidad hasta alcanzar la espiritualidad del ser humano. Considera al hombre como una unidad tridimensional, estas dimensiones son: a) la biológica, que abarca lo corporal, lo instintivo del hombre; b) la capa psíquica está constituida por la afectividad, es decir, las emociones; ambas dimensiones forman la unidad psicofísica; y c) la capa noológica comprende lo espiritual, y está formada por las facultades espirituales: inteligencia, voluntad. Utiliza el término "noodinámica" (5) para explicar que entre lo psicofísico y la fuerza espiritual se establece una lucha interna que origina un estado de tensión. Sin embargo, se mantiene la unidad porque lo espiritual domina sobre lo psicofísico. Considera que el hombre logra madurez de la personalidad cuando las tres capas funcionan armónicamente desde lo espiritual.

Afirma que la persona tiene un valor incondicional, una razón de ser basada en una misión que debe cumplir, con ella encuentra sentido a la vida. Aquellas personas cuya existencia carece de sentido viven en la mediocridad, el aburrimiento y el conformismo. Caen en lo que Frankl llama "vacío existencial".

Según Frankl, el sentido es la respuesta a las circunstancias concretas de la vida singular y única de cada individuo. La vida adquiere sentido, se plenifica, cuando se enriquece al realizar los valores de creación, de experiencia o vivenciales y de actitud.

De acuerdo con el pensamiento humanístico se atiende a la dignidad de la persona humana y se afirma que la pedagogía necesita tomar en cuenta "la conciencia, la ética, la individualidad y los valores espirituales del ser humano".

\section{FAMILIA}

La familia, "comunidad natural duradera de padres e hijos, es la 
más antigua y más íntima sociedad educativa; su importancia radica en que es una comunidad de vida corporal y psíquica; los miembros que la integran se sienten unidos entre sí por lazos de parentesco y amor".

Los auténticos cimientos de esta unión son: la libertad, el altruismo y el amor; quedan fuera el egoísmo, el miedo y la coacción. Por su flexibilidad, la familia tiene la posibilidad de adaptarse y armonizar a través del tiempo y en determinadas circunstancias sociales y culturales de la humanidad. La familia continúa siendo una comunidad de afecto espiritual y moral; en ella se da una convivencia entre personalidades abiertas, cada una a las demás 9 .

Por amor corporal y espiritual, esposo y esposa se unen para el nacimiento de los hijos. Esto significa que en la misma naturaleza del hombre y de la mujer están inscritas dos tendencias: la sexualidad y la sociabilidad, que los lleva a constituir el matrimonio sobre el cual se funda la familia ${ }^{10}$. El hombre y la mujer unidos en matrimonio adquieren el compromiso de cumplir libremente con el deber ser del esposo y el deber ser de la esposa, de convivir, procrear y educar a sus hijos; todo esto en forma racional, humana.

Educar a los hijos es lo que le da sentido a la paternidad y a la maternidad. Los padres son los primeros y principales educadores; tienen el derecho y el deber de proporcionarles la mejor educación posible. Para cumplir con esta finalidad responsablemente se requiere que estén educados, que continuamente se ocupen de su propia educación.

La familia cobra especial importancia porque es el primer ambiente natural que se requiere para la educación. La familia constituye una comunidad educativa de vida y amor donde se recibe el trato de persona y se aprende a ser mejor persona con el concurso de padres, hijos, hermanos y otros familiares. En este sentido, la responsabilidad de los padres es decisiva para la educación de los hijos. Esta función se lleva a cabo felizmen- 
te si entre los padres existe primero una relación armónica y de superación, de tal manera que les permita mantener la estabilidad de su hogar.

\section{NECESIDAD DE LA ORIENTACIÓN FAMILIAR}

Es un hecho que la mayoría de las familias enfrentan problemas educativos que impiden satisfacer necesidades legítimas de los padres. Por ejemplo: que los hijos obedezcan, que el cónyuge muestre cariño, que los adolescentes participen activamente en casa, que establezcan momentos para el descanso y la reflexión, etcétera. También observamos a padres que carecen de conocimientos necesarios acerca de lo que significa matrimonio, amor conyugal, vida de familia, así como educación integral de los hijos.

La orientación familiar ofrece contenidos, recursos y procedimientos para que padres, hijos, abuelos y otros miembros de la familia aprendan a convivir con más armonía, aumentando así la posibilidad de alcanzar las metas que se habían propuesto para desempeñar sus funciones educativas. El pedagogo está consciente de que la mayoría de las familias carecen de conocimientos y habilidades indispensables para identificar con claridad los problemas que enfrentan y contar con alternativas de solución. Por tal motivo necesitan el apoyo pedagógico a través de la orientación familiar.

\section{¿QUÉ ES LA ORIENTACIÓN FAMILIAR?}

Es la intervención pedagógica aplicada durante el ciclo vital de la familia. Se utilizan aportaciones de diversas disciplinas, a fin de guiar y aconsejar a los miembros de una familia para que sean capaces de establecer relaciones intrafamiliares e interfamiliares satisfactorias, en beneficio de cada uno de los integrantes en particular y de la sociedad en general.

Se define a la orientación familiar como "...un servicio de ayuda para la mejora personal de quienes integran una familia, 
y para la mejora de la sociedad en y desde la familia"11. Se trata de la cooperación relacionada con las funciones que se realizan en el ámbito familiar, donde padres e hijos pueden dar lo mejor de sí mismos. Se imparte a personas concretas, unidas por lazos de parentesco y de amor.

La orientación familiar está dirigida a familias en distintas etapas de su ciclo vital que necesitan una guía adecuada para actuar con eficiencia. Los padres, hijos y abuelos que reciban esta orientación tendrán mejores oportunidades de actuar con acierto y facilitar la intervención de otros educadores, quienes reforzarán la buena educación impartida, o ayudarán a rectificar los posibles errores que aquellos pudieron cometer.

Según Moratinos “...la orientación familiar implica normas preventivas para evitar anormalidades y deficiencias de la educación familiar" ${ }^{\prime 2}$. Esta intervención queda incluida en los servicios de ayuda pedagógica. Se comprende que las anormalidades y deficiencias se refieren a las personas que integran la familia, quienes por alguna incapacidad o ignorancia pueden provocar la aparición de perturbaciones emocionales en la dinámica familiar, y poner en peligro el proceso educativo de quienes conviven en este ámbito.

Al plantear la orientación familiar como un servicio de ayuda de carácter preventivo y correctivo se espera que con la guía del pedagogo, los integrantes de una familia aprovechen más los aciertos, adviertan aquellos signos que revelan posibles anomalías o actuales carencias de educación, y que sepan eliminar o contrarrestar los efectos indeseables que causaron.

\section{PROGRAMAS DE ORIENTACIÓN FAMILIAR}

Otra función del pedagogo es planificar las actividades de orientación familiar. Este trabajo exige un plan definido por "razones de responsabilidad moral, económica, adecuación laboral y eficiencia"13.

Los programas de orientación familiar se clasifican en el nivel 
de iniciación, ya que se ocupan de temas generales, cuya elección resulta difícil, debido a la cantidad de material disponible. Para resolver esta dificultad, el pedagogo toma en cuenta que los contenidos tengan un valor funcional, de aplicación práctica.

Son temas ligados a problemas de actualidad; se incluyen asuntos relacionados con los conceptos de persona, libertad, matrimonio, familia, autoridad de los padres, virtudes humanas, valores, influencia de ideologías en la familia, infancia, adolescencia, educación para el amor y muchos más. La selección obedece a intereses de padres de familia y a la necesidad de mejorar sus acciones educativas en el ámbito familiar.

Los objetivos generales del programa de orientación familiar son:

a) Distinguir los principios básicos del matrimonio y la familia

b) Aplicar estos principios en el desarrollo integral de la familia

c) Valorar la orientación familiar como medio para mejorar nuestra sociedad a partir de la familia

Con base en estos objetivos generales el pedagogo diseña los programas, cuyos objetivos particulares enunciamos a continuación:

a) Profundizar en el conocimiento de la vida familiar

b) Influir positiva y constructivamente en las relaciones familiares

c) Identificar problemas que obstaculizan el desarrollo de la familia

d) Sugerir alternativas de solución a los problemas detectados

Por medio de los programas de orientación familiar el pedagogo procura obtener resultados significativos que aumenten la calidad de los padres en su quehacer educativo. El pedagogo espera que los participantes reconozcan los beneficios obtenidos en estos programas, y que continúen su formación acu- 
diendo a los programas posteriores que ofrecen las Escuelas de Padres de Familia.

\section{DESTINATARIOS}

¿Quiénes participan? Los programas de orientación familiar están diseñados en función de los padres de familia, ya que son ellos quienes realmente se benefician con las experiencias de aprendizaje que dirige el pedagogo.

Los participantes en estos cursos son adultos de distinta procedencia familiar y sociocultural; han vivido gran cantidad de experiencias que influyen en su comportamiento. Son diferentes sus juicios y expectativas respecto al matrimonio, la paternidad, la maternidad y la familia. Todos han hecho una elección profesional y desempeñan un trabajo determinado en empresas y en hogares diferentes. Los grupos son heterogéneos, pero una característica común entre ellos es que asisten al programa de orientación familiar.

Son adultos que basan su éxito en el uso de su inteligencia y en distintas habilidades para el desempeño de su trabajo. Muestran interés por mejorar su vida familiar con responsabilidad; por tal motivo rechazan cualquier programa que suponga una mera indoctrinación o pérdida de tiempo, esfuerzo y dinero. En cambio, su rendimiento es mayor cuando el programa de orientación familiar satisface sus intereses y necesidades.

El orientador sabe que los participantes tienen anhelos insatisfechos y conflictos no resueltos en áreas familiar, social y afectiva; por eso procura que las sesiones sean de aprendizaje y resolución de problemas de casos programados; no utiliza el aula para reuniones de terapia familiar. Pero, cuando ellos la piden, se les proporciona consultoría de orientación personal, matrimonial y familiar.

Desde el punto de vista social, quienes asisten al curso de orientación familiar piensan y buscan las relaciones interpersonales. Esperan respeto y aceptación incondicional en 
el trato ${ }^{14}$. Por esto el pedagogo dirige el trabajo en grupo mediante una metodología facilitadora de la interacción e interrelación de los participantes, quienes al aumentar su confianza logran aportaciones que enriquecen al grupo.

Como adultos conocen sus cualidades y limitaciones. Son conscientes de los valores que orientan su conducta. Piensan en su autorrealización. Esperan ser reconocidos por sus valiosas aportaciones y disculpados por sus errores en la participación grupal.

En cuanto a sus valores, los participante han establecido una jerarquización respecto a lo religioso, lo moral, lo estético, lo intelectual, etcétera. Pueden aceptar o rechazar ideas expresadas durante las sesiones. En estos casos el pedagogo no impone sus criterios, manifiesta su flexibilidad intelectual, respeta lo que piensa cada participante y evita discusiones innecesarias. Algunos participantes afirman que han cambiado su manera de pensar cuando escucharon ideas convincentes acerca del sentido de la vida.

Contribuir a la orientación familiar de adultos es una tarea que fatiga, pero resulta muy gratificante por el efecto multiplicador que esto origina; ellos se convierten en transmisores de alegría y felicidad en su familia y en la comunidad.

\section{METODOLOGÍA PARTICIPATIVA}

La orientación familiar no tiene una metodología cerrada. Sugiere diversas opciones para lograr un clima de confianza, aceptación y respeto en las sesiones El propósito es que los participantes tengan confianza en sí mismos, sientan el gusto y la libertad de aprender ${ }^{15}$. En las actividades con padres de familia se pretende conseguir un cambio de actitudes y que aprendan a reflexionar en la toma de decisiones.

En general, los participantes han dejado de estudiar formalmente; sus hábitos de trabajo intelectual son regulares por falta de práctica . Esta es una razón por la cual prefieren un método 
menos académico. La mayoría de ellos responde mejor al método inductivo que al deductivo. Se utilizan técnicas de dinámica grupal para que los participantes mantengan el interés, y el pedagogo no abuse de la técnica expositiva.

Una característica de la metodología participativa es que cada persona al dejar su postura expectante de escuchar, mirar y esperar, adquiere otra de participación activa en la situación de aprendizaje, involucrándose como una parte de ella. También es indispensable desarrollar la capacidad de estudio individual para contribuir con el grupo a lograr objetivos educativos, ya que las aportaciones bien elaboradas de cada uno enriquecen la sesión.

La metodología participativa facilita la comunicación en distintas direcciones y favorece el perfeccionamiento de los padres de familia. Con la ayuda del pedagogo se crea un clima educativo y de reflexión para abordar los temas relacionados con la orientación familiar. Esta metodología incluye técnicas que promueven la dinámica grupal, de tal manera que el aprendizaje sólo se logra como producto de la actividad efectiva de los participantes.

El orientador acepta las diferentes intervenciones, procura que sean breves, relaciona unas con otras, destaca lo más importante, les pide que hagan síntesis frecuentes, formula preguntas para promover el pensamiento reflexivo; aporta información necesaria para la comprensión de los temas, y tiene en mente los objetivos de aprendizaje, así como los intereses del grupo $^{16}$.

Otra característica de la metodología participativa es que a pesar de lo planeado, se corre el riesgo de lo sorpresivo, ya que la participación es impredecible. El pedagogo desconoce hacia dónde se dirigirá el comportamiento de los participantes. El riesgo está en que tal vez la participación efectiva no se produzca; o que los intereses del grupo se orienten hacia objetivos que no estaban previstos, pero que satisface necesidades 
más inmediatas de los participantes, sin descuidar los contenidos programados.

La combinación de técnicas, materiales, procedimientos y estilos de dirección hacen de la metodología participativa, un sistema complejo que presenta algunas dificultades superables.

Estas dificultades se deben a varios factores; entre otros: algunos participantes reflexionan muy bien, pero temen expresar sus pensamientos. Otros carecen de la habilidad para analizar una situación compleja, ya sea en el método del caso o en la profundización de notas técnicas. A veces ocurre que el grupo se resiste a cambiar su actitud pasiva por un trabajo más dinámico y creativo.

Ante estos problemas el pedagogo adopta una actitud de comprensión y paciencia. Pero en forma progresiva, y a la brevedad posible, orienta al grupo en la aplicación de esta metodología, ya que es un sistema indispensable para trabajar con padres de familia, para entrenarlos en nuevos modos de pensar y actuar, y para que en sus respectivas familias exista un ambiente donde padres e hijos participen productivamente.

\section{EVALUACIÓN DEL PROGRAMA DE ORIENTACIÓN FAMILIAR}

El pedagogo interviene en todos los momentos del proceso didáctico: planeación, realización y evaluación del programa de orientación familiar en general, y de las sesiones de trabajo en particular.

La realización de un programa supone aprendizaje, el cual implica un cambio en la manera de pensar, sentir o actuar. Por tal motivo es necesario evaluar para conocer si lo que planeamos se cumplió o no, y por qué. "La evaluación espera conocer y valorar no sólo los resultados conseguidos, sino también la correlación que existe entre estos y los medios utilizados"17. Además se necesita para justificar que el esfuerzo valió la pena por los beneficios que reditúa a los participantes y a sus familias. 
En los programas de orientación familiar, el pedagogo utiliza la "evaluación de la reacción"18. Consiste en aplicar un cuestionario para obtener datos acerca del grado en que la sesión gustó o no a los participantes. Reporta el agrado o desagrado por la forma de llevarse a cabo el programa, más que el aprendizaje formal de los contenidos temáticos.

Sin embargo, se pretende obtener alguna información acerca de aprendizajes significativos en cada sesión respecto al contenido captado por cada participante, en función de los objetivos propuestos. En el cuestionario de evaluación se incluye una pregunta de respuesta abierta para este propósito

Las demás respuestas del participante revelan el éxito del programa debido a la impresión causada por el pedagogo, lo novedoso de los temas, las técnicas de trabajo en grupo, el ambiente de cordialidad, etcétera. Todo esto se refiere a la respuesta afectiva, más que la de efectividad.

Al terminar cada programa, el pedagogo aplica la evaluación final. El propósito es conocer, en cierto modo, cómo ha influido el contenido del programa en la vida personal y familiar de los participantes. Se evalúa la reacción de ellos ante todo el programa. Para esta evaluación se aplica un cuestionario de respuesta abierta. Se les pide que respondan a estas preguntas. ¿Cuál es tu opinión acerca de este programa?, ¿Qué otros temas deberían ser tratados?, ¿Qué sugieres para mejorar el programa? También se les pregunta acerca de los cambios personales y familiares originados por la influencia del programa de orientación familiar.

\section{CONCLUSIÓN}

Hemos mencionado que las familias se ven afectadas por los cambios vertiginosos que ocurren en nuestra sociedad. Los niños y adolescentes manifiestan problemas de obediencia, bajo rendimiento escolar, dificultad en sus relaciones interpersonales... Los cónyuges experimentan conflictos maritales y confusión de 
sus papeles como padre y madre. Además, esta situación repercute en el rendimiento laboral y doméstico de los padres de familia, por problemas emocionales que no logran resolver satisfactoriamente.

Nuestra experiencia con grupos de padres de familia, en diversas circunstancias, nos ha permitido comprobar que la orientación familiar, como intervención pedagógica, es efectiva para producir cambios en el comportamiento, el pensamiento y la afectividad de los participantes.

Afirmamos que esta intervención es principalmente preventiva; es decir, se trata de crear un ambiente familiar donde todos unan sus esfuerzos para afrontar los problemas que surjan. Pero también es correctiva; significa prepararse para actuar en situaciones críticas, productoras de tensiones por errores cometidos, que cuentan con el apoyo eficaz y eficiente de un pedagogo dedicado a la orientación familiar.

\section{REFERENCIAS BIBLIOGRÁFICAS}

${ }^{1}$ MAGAZ ,C. Familia hoy y mañana. Mensajero. España. 1988.

${ }^{2}$ Cfr. RIOS, J.A. Crisis familiares: causas y repercusiones. Narcea. Madrid. 1983.

${ }^{3}$ DICARPIO. N. S. Teorías de la personalidad. Interamericana. México, 1985, p 279.

${ }^{4}$ Cfr. ROGERS, C.R. Psicoterapia centrada en el cliente. Paidós. México. 1990.

${ }^{5}$ FRANKL, V. El hombre en busca de sentido. Herder. Barcelona, 1993, p. 103.

${ }^{6}$ FRANKL, V. op. cit., p. 105.

${ }^{7}$ GUZMÁN, J.C., GARCÍA, H. y HERNÁNDEZ, G. Las teorías de la psicología educativa. Análisis por dimensiones educativas. Facultad de Psicología. UNAM, 1991, p. 17.

${ }^{8}$ GÓMEZ, P.R. Familias en paz. Alba. México, 1985, p. 14.

${ }^{9}$ Cfr. GÓMEZ, P.R. op. cit.

${ }^{10} \mathrm{Cfr}$. PACHECO, A. La familia en el derecho civil mexicano. Panorama. México. 1985

${ }^{11}$ OTERO, O.F. ¿Qué es la orientación familiar? EUNSA Pamplona,1989, p. 17. 
${ }^{12}$ MORATINOS, J.F. Escuela de padres: Educación familiar. Narcea. Madrid, 1985 , p. 17.

${ }^{13}$ NÉRICI, I. Hacia una didáctica general dinámica. Kapelusz. México, 1990, p. 135.

${ }^{14}$ Cfr. ROGERS, C.R. op.cit.

${ }^{15}$ Cfr. GUZMÁN, J.C., GARCÍA, H. y HERNÁNDEZ, G.

${ }_{16}$ Cfr. VÁZQUEZ, G. G. El perfeccionamiento de los profesores y la metodología participativa. EUNSA. Pamplona. 1976.

${ }^{17}$ REZA, J.C. ¿Cómo desarrollar y evaluar programas de capacitación en las organizaciones. Panorama. México, p. 99.

${ }^{18}$ Cfr. REZA, J. C. op. cit. 